\title{
Percutaneous Gastrostomy Placement for Decompression of the Excluded Gastric Remnant following Roux-en-Y Gastric Bypass Surgery
}

\author{
Shamaita Majumdar ${ }^{1}$ Tatulya Tiwari ${ }^{1}$ Olaguoke Akinwande ${ }^{1} \quad$ Raja Siva Ramaswamy ${ }^{1}$ \\ ${ }^{1}$ Mallinckrodt Institute of Radiology, Washington University in \\ St. Louis, Saint Louis, Missouri, United States

\begin{abstract}
Address for correspondence Raja Ramaswamy, MD, Mallinckrodt Institute of Radiology, Washington University in St. Louis, $510 \mathrm{~S}$ Kingshighway Blvd, CB 8131, Saint Louis, MO 63110, United States (e-mail: ramaswamyr@wustl.edu).
\end{abstract}

J Clin Interv Radiol ISVIR 2018;2:144-148

\begin{abstract}
Purpose To evaluate the feasibility and safety of percutaneous gastrostomy for decompression of the excluded stomach in patients' status post Roux-en-Y gastric bypass (RYGB).

Materials and Methods Between January 2001 and August 2017, 10 consecutive RYGB patients who underwent placement of a decompressive gastrostomy of the excluded stomach were identified in an institutional database. Technical success was defined as successful gastrostomy catheter placement in the bypassed stomach using fluoroscopy and/or ultrasound guidance. Clinical success was established if dilation of the excluded stomach improved after gastrostomy with resolution of associated symptoms. Charts were reviewed for treatment-related adverse events post-procedure.

Results The cohort was predominantly female (9/10), with an average age of $54 \pm 14$ years. Median follow-up was 35.2 months (range: $0.6-115$ ). Indications for decompressive gastrostomy placement included small bowel obstruction (6/10) or afferent limb obstruction at the jejunojejunal anastomosis (4/10). The most common presenting symptoms were abdominal pain, distension, and vomiting. All patients had successful gastrostomy placement in the excluded remnant, using ultrasound and fluoroscopic guidance, with no procedural complications. The 12 to $16 \mathrm{~F}$ Cope loop catheters was used in this cohort, and gastropexy sutures were used in two cases. All 10 patients demonstrated clinical resolution of symptoms after gastrostomy place-

Keywords

- Roux-en-Y

- percutaneous decompression

- gastrostomy ment. Two patients developed minor complications of tube site leakage and poor tube function requiring gastrostomy exchange within 1 week of the procedure.

Conclusion Fluoroscopic and ultrasound-guided percutaneous gastrostomy catheter placement is a safe, effective, and feasible approach to treating dilation of the excluded gastric remnant in RYGB patients.
\end{abstract}

RouX-en-Y gastric bypass (RYGB) has been shown to result in durable weight loss in morbidly obese patients. ${ }^{1}$ This procedure creates a small gastric pouch that restricts food consumption. RYGB also limits the extent of nutrient absorption, as it excludes the gastric body, antrum, duodenum, and proximal jejunum. ${ }^{1,2}$ The excluded stomach remains in continuity distally with the remaining gastrointestinal tract via the afferent pancreaticobiliary limb of reconstruction, which is anastomosed distally to form a side-to-side jejunojejunostomy. Complications following RYGB are common and can include postoperative ileus, late distal small bowel obstruction (SBO), or mechanical obstruction at the jejunojejunal anastomosis (defined here as afferent loop obstruction [ALO]). ${ }^{3,4}$ These received

August 16, 2018 accepted after revision

September 19, 2018

published online

December 4, 2018
Dol https://doi.org/

$10.1055 / \mathrm{s}-0038-1676197$

ISSN 2457-0214.
(C) 2018 by Indian Society of

Vascular and Interventional Radiology
License terms

(요 (1) $\Theta \circledast$ 
complications in particular can lead to distention of the excluded portion of stomach and even lead to risk of perforation. Traditionally, treatment for distal SBO includes nasogastric tube placement for decompression and bowel rest, versus immediate surgical exploration for patients who appear septic, unstable, or peritonitic on abdominal examination. Specifically for patients with ALO, dilation of the stenotic anastomosis site with single-balloon enteroscopy can be performed as the first-line therapy in addition to decompression. ${ }^{5}$ However, the anatomic reconfiguration established by RYGB often precludes antegrade access to the bypassed stomach and bowel, including placement of a nasogastric tube for decompression. ${ }^{2,4}$ In such cases, imageguided percutaneous gastrostomy may be performed to decompress the dilated remnant stomach. The incidence of remnant stomach distention in RYGB patients is not clear. One prospective study of 246 RYGB patients reported a $7.3 \%$ rate of postoperative intestinal obstruction, with 3 cases secondary to jejunojejunostomy stricture (1.2\%). ${ }^{6}$

Successful computed tomography (CT)-guided percutaneous gastrostomy for decompression of the distended excluded segment after bariatric surgery has been previously described in small case series reports by Stein et al and Nosher et al, but there has not been any literature describing decompression gastrostomy after bariatric surgery using only fluoroscopy and ultrasound (US) guidance. ${ }^{2,4}$ The avoidance of CT and use of US and limited fluoroscopy guidance instead may help reduce radiation exposure in these patients. Additionally, Nosher et al describe using a combination of CT, fluoroscopy, and US guidance in several of the cases in the study, potentially reducing efficiency by relying on so many modalities during a single procedure. Last, percutaneous gastrostomy for decompression in this subset of patients may be a safe and feasible alternative to surgery that mitigates the risks of morbidity and mortality associated with open surgical decompression. In this series, we describe our experience with 10 consecutive cases of percutaneous gastrostomy placement for decompression of the excluded gastric remnant, using only US and fluoroscopic guidance. The purpose of this article is to evaluate the feasibility and safety of percutaneous gastrostomy for decompression of the excluded stomach in patients status post RYGB.

\section{Materials and Methods}

This study was designed as an institutional review board (IRB)-approved (IRB \#201806190) retrospective case series. Departmental records from a prospectively maintained database were reviewed to identify cases of percutaneous gastrostomy performed between January 2001 and August 2017. Inclusion criteria were as follows: (1) The patient underwent prior RYGB, (2) the target for tube placement was the excluded gastric remnant, and (3) the indication for procedure was ileus, distal bowel obstruction, or ALO with dilation of the excluded segment requiring decompression. Prior to their inpatient referral to interventional radiology for treatment, patients were typically diagnosed by their primary medical teams using a combination of clinical symptoms indicative of obstruction (nausea, vomiting, bloated, distended abdomen, absent signs of bowel function) and ancillary CT imaging that would reveal dilation of the excluded gastric lumen, often along with a transition point (for SBO) or stenosis at the anastomotic site (for ALO). Eight interventional radiologists at our institution with an average of 15 years of operator experience were involved with managing the procedures in this cohort. Technical success was defined as successful placement of a gastrostomy catheter in the bypassed portion of stomach. The follow-up period was defined as the time point from initial catheter placement until the date of chart review. To establish clinical success post-procedure, patient charts were retrospectively interrogated for documentation of clinical resolution of obstructive symptoms within the follow-up period. Additional follow-up outcomes included time point of catheter removal and any treatment-related adverse events at any point post-procedure within the follow-up period.

Informed consent to undergo percutaneous gastrostomy was obtained from all patients prior to each procedure following a discussion of risks, benefits, and alternatives. Preprocedurally, relevant laboratory parameters and available imaging were reviewed ( - Fig. 1A). All procedures were performed under moderation sedation. The skin over the stomach was sterilely prepped, draped, and infiltrated with $1 \%$ lidocaine. The upper abdomen was carefully examined both with fluoroscopy and US ( - Fig. 1B). The decision to perform gastropexy
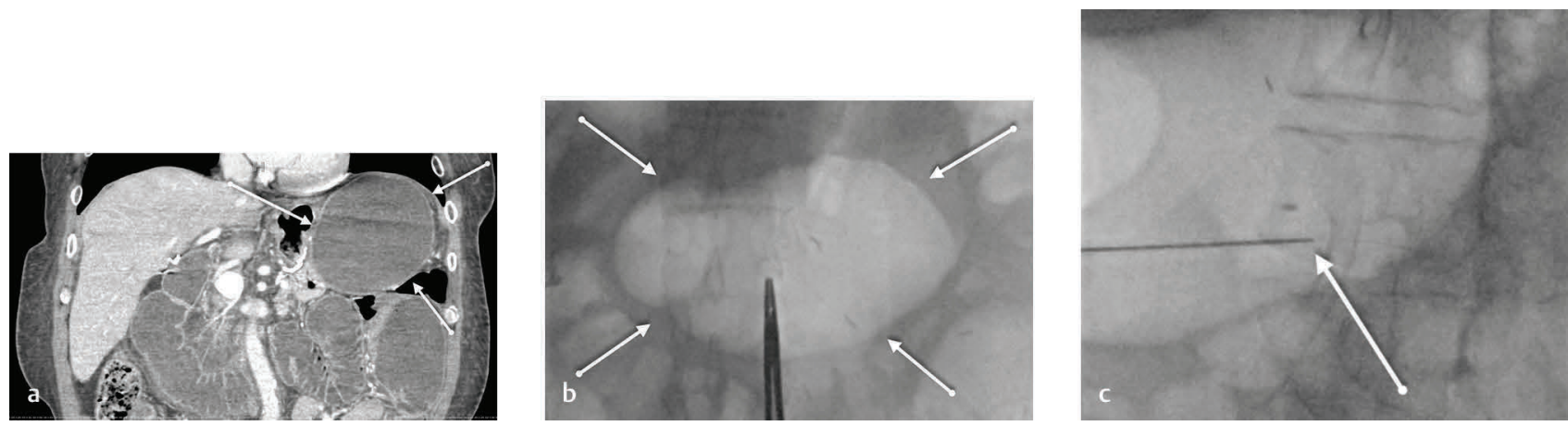

Fig. 1 A 64-year-old man with history of RYGB in 2003 who presented in 2016 with abdominal pain. CT scan revealed obstruction of the afferent limb along with a massively dilated excluded gastric remnant in the coronal view (white arrows) (A). The patient was brought to interventional radiology for decompression of the gastric remnant. The upper abdomen was carefully examined both with fluoroscopy and ultrasound, and the targeted stomach was noted to be massively dilated under fluoroscopy (white arrows) (B). Two gastropexy sutures were placed, and then the targeted distended stomach was accessed with a 19 gauge single-wall needle (white arrow) (C). 
was left to the discretion of the individual practitioners in a case-by-case basis, and gastropexy sutures were used for two of the patients in this cohort. In patients who underwent gastropexy, two T-fasteners were placed into the anterior wall of the distal gastric remnant under US guidance and sutured to the skin. US and fluoroscopy modalities were used to identify a safe access target on the excluded stomach. In all cases, there were gas and fluid within the excluded stomach lumen. Fluoroscopy was used to identify the target viscera given the partially air-filled lumen. US was primarily used to visualize a point along the gastric wall that would be accessible without damaging adjacent structures such as organs or blood vessels. Using US and fluoroscopy guidance, the targeted distended stomach was accessed with a 19-gauge single-wall needle ( - Fig. $1 \mathrm{C}$ ). In each case, the specific approach with US versus fluoroscopy guidance for needle access to the excluded stomach was left to the discretion of the provider, and in this series, all providers used a combination of both techniques to ensure safe access. Contrast was injected to confirm needle placement into the gastric remnant. A 0.035in Bentson guidewire (Cook Medical) was advanced into the stomach through the needle and coiled within the stomach cavity. The tract was serially dilated over the guidewire to the appropriate diameter based on catheter size. A locking pigtail 14F multipurpose drainage catheter (Cook Medical) was advanced over the wire into the stomach, and contrast was injected through the catheter to confirm appropriate positioning within the excluded stomach $(\boldsymbol{-}$ Fig. 2). The catheter was secured to the skin using 0 silk suture and connected to gravity drainage or bulb suction. Patients were monitored for 24 hours following tube placement for signs of peritonitis or other immediate complications. If gastropexy sutures were placed, they were subsequently removed after 7 days.

\section{Results}

Ten consecutive patients underwent percutaneous gastrostomy catheter placement for decompression of the excluded bypass remnant. In this study cohort, patients presented with signs of distension or obstruction at minimum 1 year after their initial RYGB surgery. This cohort was predominantly female $9 / 10$ (90\%), with an average age of $54 \pm 14$ years (range: 28-70). Median follow-up was 35.2 months after initial catheter placement (range: 0.6-115). Technical success rate for decompressive gastrostomy placement into the gastric remnant was $100 \%$ using US and fluoroscopy (-Table $\mathbf{1}$ ). In 2 of the 10 patients, a $12 \mathrm{~F}$ catheter was placed. In seven cases, a $14 \mathrm{~F}$ catheter was initially placed, although in one of these cases, the catheter was upsized to $18 \mathrm{~F}$ due to poor function. In one case, a $16 \mathrm{~F}$ catheter was used. Two of the 10 patients had gastropexy sutures placed during the procedure. All patients ultimately demonstrated improvement in gastric remnant distention and resolved clinical symptoms following catheter placement. In four patients, tubes were removed after 8 weeks, and patients demonstrated complete symptom

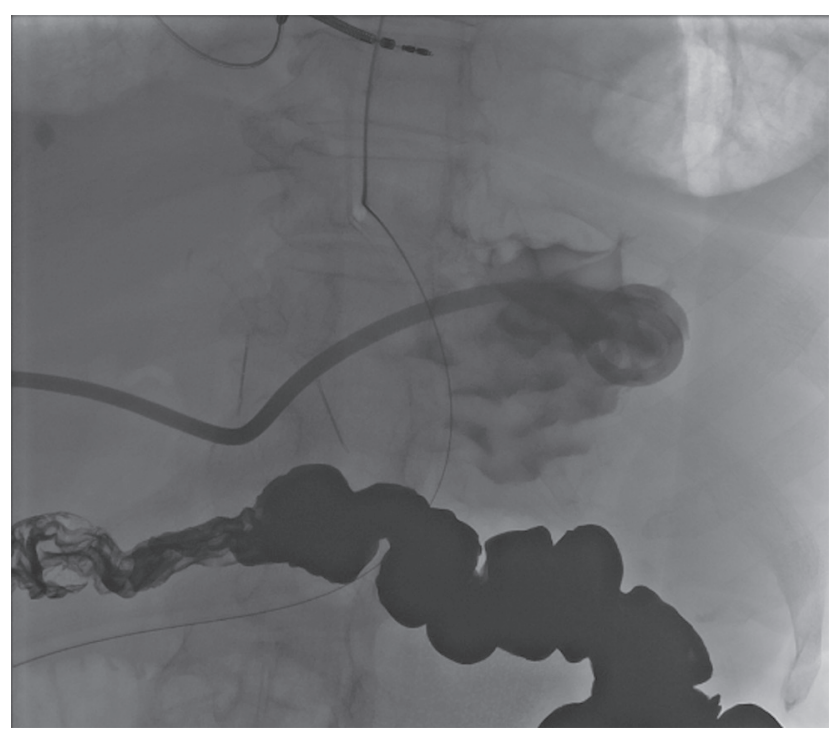

Fig. 2 A 67-year-old woman with recurrent SBO status post RYGB many years prior. Final fluoroscopic image with contrast injection via the $14 \mathrm{~F}$ catheter demonstrates the gastrostomy to be within the excluded gastric remnant.

Table 1 Procedural data for patients who received US/fluoroscopy-guided percutaneous gastrostomy into a dilated excluded gastric segment after RYGB

\begin{tabular}{|l|l|l|l|l|l|l|}
\hline Case & Age $(\mathrm{y})$ & Sex & Indication & Catheter size (F) & Gastropexy & Complications \\
\hline 1 & 67 & F & SBO & 14 & Yes & None \\
\hline 2 & 64 & M & ALO & 14 & No & None \\
\hline 3 & 47 & F & SBO & 12 & No & None \\
\hline 4 & 49 & F & SBO & 18 & Yes & Poor output; leaking \\
\hline 5 & 63 & F & ALO & 14 & No & None \\
\hline 6 & 55 & F & ALO & 14 & No & None \\
\hline 7 & 70 & F & ALO & 12 & No & None \\
\hline 8 & 28 & F & SBO & 14 & No & Poor output; leaking \\
\hline 9 & 36 & F & SBO & 16 & No & None \\
\hline 10 & 63 & F & SBO & 14 & No & \\
\hline
\end{tabular}

Abbreviations: ALO, afferent limb obstruction; F, female; M, male; RVGB, Roux-en-Y gastric bypass; SBO, small bowel obstruction; US, ultrasound. 
resolution over that period. In four patients, catheter placement resulted in clinical resolution of symptoms but was not removed until they underwent definitive surgical revision of their jejunojejunal anastomotic site or surgical lysis of adhesions as necessary for SBO. In two patients, the catheters remained in place long term, with transfer to hospice due to multiple other comorbidities.

Two patients in this cohort developed complications: one major that required rehospitalization and one minor that required catheter exchange. One patient developed recurrent distal SBO and dilation of the excluded remnant stomach after initial catheter placement (14F), and the catheter had to be upsized to $18 \mathrm{~F} 8$ days later. This patient had complete resolution of clinical SBO symptoms over the course of several days. They were ultimately transferred to hospice due to multiple medical comorbidities, and the gastrostomy tube remained in place long term for feeding. The second complication was in a patient who had pericatheter leakage around their $16 \mathrm{~F}$ tube that necessitated exchange 4 days later to another $16 \mathrm{~F}$ catheter. Following exchange, the catheter functioned appropriately. This patient's tube remained in place for several weeks with successful decompression and symptomatic improvement. Subsequently, the patient underwent surgical removal of tube at the time of their anastomotic revision.

\section{Discussion}

Distention and dilation of the excluded gastric remnant is a known complication of RYGB surgery, which clinically manifests as ileus or SBO.,4 As aforementioned, the traditional approach to treating distal SBO includes conservative supportive measures, including nasogastric tube placement, bowel rest, and intravenous fluid administration, versus immediate surgical exploration for patients who appear septic, unstable, or peritonitic on abdominal examination with concern for perforation. Specifically for patients with ALO, dilation of the stenotic jejunojejunal anastomosis site with single-balloon enteroscopy in addition to decompression is considered the first-line treatment for anastomotic strictures. ${ }^{5}$ However, due to the anatomic changes in patients status post RYGB surgery, antegrade access to the excluded stomach with nasogastric tube is often precluded, and alternative approaches must be used to access and decompress the excluded stomach. Placement of gastrostomy tubes in the excluded stomach at the time of gastric bypass surgery as a prophylactic measure against distension has been described. ${ }^{7}$ However, later studies demonstrated that routine gastrostomy at the time of gastric bypass does not improve clinical outcomes and is beneficial only in the subset of patients considered at high risk for developing obstruction or anastomotic leak. ${ }^{8}$ Additionally, these prophylactic gastrostomy tubes were typically removed after several weeks, when the risk of acute distension was lower. In our study population, most patients presented with signs of distension or obstruction at minimum 1 year after their initial gastric bypass surgery, rather than within a few weeks. Other techniques have been suggested to address risk of distension in this patient population, including the placement of radiopaque markers at the anatomical site of gastrostomy insertion, which is thought to facilitate future catheter placement if required. ${ }^{6}$

This study findings concur with two previously published retrospective case series reports endorsing the feasibility of gastrostomy catheter placement for decompression into the excluded stomach after RYGBB., ${ }^{2,4}$ In a 2007 retrospective case series, Stein et al reported on the use of CT guidance alone to place gastrostomy catheters in 10 patients. ${ }^{2}$ They reported technical success rate of $100 \%$, stating that even with only 3 patients having clear access windows, gastrostomy catheters were successfully placed under $\mathrm{CT}$ guidance in all 10 patients. One patient in the study by Stein et al had a technical complication reported: During initial access, it became evident that the gastric wall rather than the lumen was entered and was dissected during air insufflation. The procedure was aborted and reattempted a few days later after injected air could be resorbed, with successful catheter placement into the gastric lumen upon second attempt. Another retrospective study, by Nosher et al, reported on the use of a combination of CT, US, and fluoroscopy guidance to place gastrostomy catheters in a series of eight patients. ${ }^{4}$ In their study, multiple cases were started in CT and then transferred to fluoroscopy for final catheter placement. They also reported a technical success rate of $100 \%$ for percutaneous gastrostomy placement. In their series, seven of eight patients experienced clinical resolution of symptoms following catheter placement. They reported two complications, including one patient with periprocedural peritonitis with underlying SBO who ultimately required surgical intervention, and one wound infection treated with antibiotics and local wound care. In our study, US and fluoroscopy were the only modalities used for catheter placement, with a $100 \%$ technical and clinical success rate.

Overall it appears that the evidence supporting use of gastropexy in routine percutaneous gastrostomy is inconclusive. Opponents of T-fasteners assert that their use may induce tension on the gastric wall, which could lead to ischemia, necrosis, and subsequent leakage around the tube. ${ }^{9}$ However, advocates of T-tack usage maintain that tract maturation may occur more rapidly with gastropexy and consequently reduce the risk of peritonitis. ${ }^{10}$ Nosher et al describe the placement of gastropexy sutures in seven of eight cases in their previously mentioned report. ${ }^{4}$ They contend that there was concern for potential leakage from the gastrostomy because all patients had gastric distention with the ileus or obstruction and thus predominantly used T-fasteners. In their study, one of the seven patients who underwent gastropexy during the procedure subsequently developed peritonitis. In our study, use of gastropexy was left to the practitioner's discretion, and in this cohort, gastropexy sutures were used in 2 of 10 patients. One of these two patients later presented with leakage at the catheter entry site requiring subsequent upsizing, which led to resolution of the leakage. Consistent with our institutional practice, we do not have strong recommendations for or against the use of T-fasteners in this subset of patients and believe it should be left to the operator's discretion. 
Inherently this study is limited by its small sample size and its design as a retrospective case series. Because of the low incidence of this disease process, which is restrained to the RYGB population, large prospective cohort studies and randomized controlled trials would be extremely difficult to carry out. Despite such limitations, we maintain with cautious optimism that fluoroscopic- and US-guided decompressive gastrostomy is a reasonable approach for this subset of patients. Our rate of successful decompression is corroborated by high rates of technical success described in the limited published data.,4 The existing literature affirms that effective image-guided percutaneous gastrostomy eliminates the need for prophylactic placement of a surgical gastrostomy at the time of gastric bypass surgery to prevent gastric distension as a complication. ${ }^{4,8}$ Additionally, we assert based on our own technique that percutaneous gastrostomy of the excluded remnant stomach can be performed efficaciously without requiring or relying on previously placed radiopaque markers.

While traditional percutaneous gastrostomy tubes in non-RYGB patients can typically be performed with fluoroscopy alone, using insufflation of air into the stomach via nasogastric tube and subsequent visualization of the target viscera on fluoroscopy, antegrade access to the excluded stomach is often precluded due to post-surgical anatomic changes in RYGB, and a nasogastric tube cannot be placed. Therefore, multiple imaging modalities are often required to aid visualization and avoid complications such as injury to the bowel, blood vessels, and other surrounding organs. In this study cohort, all cases involved gastric lumens that were distended with both air and fluid. There was heavy reliance on the use of US to identify and avoid important adjacent structures such as bowel or blood vessels along the gastric wall. Fluoroscopy was primarily used to identify our target viscera using air already within the lumen of the excluded stomach. We concede that access of the excluded stomach is considerably more difficult when the distended stomach is not partially air-filled and thus requires careful sonographic evaluation. However, if there is no appropriate visualization, we advocate for the use of CT guidance if a safe access window cannot be appreciated using US and/or fluoroscopy.

Complications following RYGB are common and can include postoperative ileus, late distal SBO, or mechanical obstruction at the jejunojejunal anastomosis. These complications in particular can lead to distention of the excluded portion of stomach that remains in continuity distally with the remaining gastrointestinal tract. Traditional imageguided approaches to percutaneous gastrostomy with fluoroscopy alone are difficult due to poor visualization of the excluded stomach using a single imaging modality. Whereas earlier studies have reported the feasibility of using CT guidance alone or a combination of CT with fluoroscopy and US guidance in this subset of patients, there are no reports prior to this study describing the use of fluoroscopy and US alone to guide percutaneous gastrostomy in patients status post RYGB. When appropriate visualization with these two imaging modalities can be achieved in this setting, the avoidance of CT may help reduce radiation exposure in these patients. Our retrospective analysis in this case series indicates that fluoroscopic- and US-guided percutaneous gastrostomy is a safe, effective, and feasible approach to treating dilation of the excluded gastric remnant in RYGB patients.

\section{Conflict of Interest}

The authors declare that they have no conflict of interest.

\section{Acknowledgments}

None.

\section{References}

1 Colquitt JL, Pickett K, Loveman E, Frampton GK. Surgery for weight loss in adults. Cochrane Database Syst Rev 2014;(8):CD003641.

2 Stein EG, Cynamon J, Katzman MJ, et al. Percutaneous gastrostomy of the excluded gastric segment after Roux-en-Y gastric bypass surgery. J Vasc Interv Radiol 2007;18(7):914-919

3 See C, Carter PL, Elliott D, et al. An institutional experience with laparoscopic gastric bypass complications seen in the first year compared with open gastric bypass complications during the same period. Am J Surg 2002;183(5):533-538

4 Nosher JL, Bodner LJ, Girgis WS, Brolin R, Siegel RL, Gribbin C. Percutaneous gastrostomy for treating dilatation of the bypassed stomach after bariatric surgery for morbid obesity. AJR Am J Roentgenol 2004;183(5):1431-1435

5 Siddique SS, Feuerstein JD. Gastrointestinal complications of Roux-en-Y gastric bypass surgery. OA Minim Invasive Surg 2014;2(1):1-8

6 Papasavas PK, Caushaj PF, McCormick JT, et al. Laparoscopic management of complications following laparoscopic Roux-en-Y gastric bypass for morbid obesity. Surg Endosc 2003;17(4):610-614

7 Fobi MA, Chicola K, Lee $\mathrm{H}$. Access to the bypassed stomach after gastric bypass. Obes Surg 1998;8(3):289-295

8 Wood MF, Sapala JA, Sapala MA, Schuhknecht MP, Flake TM. Micropouch gastric bypass: indications for gastrostomy tube placement in the bypassed stomach. Obes Surg 2000;10(5):413-419

9 Dewald CL, Hiette PO, Sewall LE, Fredenberg PG, Palestrant AM. Percutaneous gastrostomy and gastrojejunostomy with gastropexy: experience in 701 procedures. Clin Otolaryngol 2009;34:103-112

10 de Baere T, Chapot R, Kuoch V, et al. Percutaneous gastrostomy with fluoroscopic guidance: single-center experience in 500 consecutive cancer patients. Radiology 1999;210(3):651-654 